\title{
AFM-Téléthon Congrès Myology 2016 : cinq jours d'une rare intensité
}

\author{
Tuy Nga Brignol, Gaëlle Barrier, J. Anndoni Urtizberea
}

La $5^{\mathrm{e}}$ édition du congrès international de Myologie organisée par l'AFM-Téléthon s'est déroulée au Palais des congrès de Lyon, du 14 au 18 mars derniers, en présence des plus grands experts mondiaux de la discipline. Un rassemblement marqué par des avancées thérapeutiques pour les maladies neuromusculaires, la présentation des résultats d'essais cliniques et les premiers enseignements tirés de ces essais.

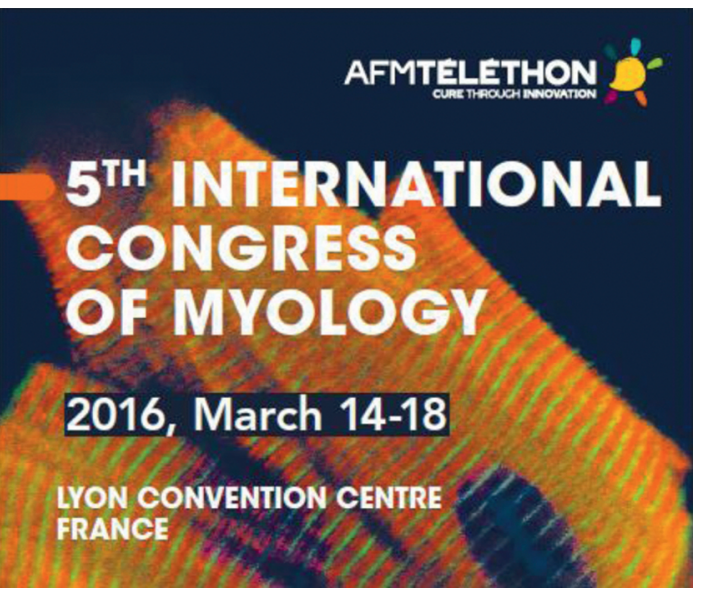

Cinq ans après le dernier congrès dédié à la myologie ayant eu lieu à Lille en 2011, la nouvelle édition du congrès Myology à Lyon a rassemblé 982 participants (experts, chercheurs, patients, industriels). Quelques chiffres clés: 420 posters, 10 séances plénières, 14 symposia, 6 conférences satellites, 1 séance plénière du conseil scientifique de l'AFM-Téléthon.

La $1^{\text {re }}$ journée a été marquée par la conférence inaugurale de Kenneth Fischbeck (Bethesda, USA), l'un des signataires en 1986 de la publication annonçant la découverte du gène $D M D$ responsable de la myopathie de Duchenne (NB: la dystrophine, c'est l'année d'après).

Lors de la $2^{e}$ journée, les sessions plénières ont été consacrées à la biologie du muscle et à la jonction neuromusculaire. Christophe Marcelle (Lyon), précédemment responsable d'une équipe de recherche à l'Australian Regenerative Medicine Institute de l'Université Monash (Melbourne) a ouvert la journée. Le projet qu'il mène depuis 2009 sur la formation, le développement et la réparation du muscle se poursuit aujourd'hui au sein du tout nouvel Institut NeuroMyoGène à Lyon, en étroite interaction avec l'Alliance MyoNeurALP sur les maladies neuromusculaires en Auvergne-Rhône-Alpes.

La $3^{e}$ journée a débuté par une session plénière consacrée au développement des thérapies innovantes. Matthew Wood (Université d'Oxford, UK) a présenté ses travaux sur la restauration de l'expression de la dystrophine chez la souris $m d x$ grâce à des peptides de nouvelle génération améliorant le saut d'exon, et permettant de cibler plus efficacement un grand nombre de tissus de l'organisme, notamment le cœur et le système nerveux central. Aurélie Goyenvalle (Université de Versailles SaintQuentin-en-Yvelines) a développé une approche de pharmaco-génomique utilisant un nouveau type d'oligonucléotide antisens qui a été testé avec succès chez la souris. Caroline Le Guiner (Atlantic Gene Therapies, Nantes) a présenté les résultats de ses travaux de thérapie génique utilisant un vecteur AAV sur le chien GRMD. Dans la session plénière de l'après-midi, les intervenants ont présenté plusieurs traitements pharmacologiques potentiels pour diverses maladies neuromusculaires: tamoxifène dans la myopathie de Duchenne (Olivier Dorchies, Suisse), tréhalose dans la dystrophie musculaire oculo-pharyngée (Zohar Argov, Israël), ManNAc dans la myopathie GNE (Ichizo Nishino, Japon) et $\mathrm{N}$-Acétylcystéine dans la myopathie liée à la sélénoprotéine N (Ana Ferreiro, Université Paris Diderot). La $4{ }^{e}$ journée du congrès s'est ouverte sur une session plénière dédiée aux différentes avancées en Myologie. Lee Sweeney (Floride, USA), co-président du congrès, a fait le panorama des essais cliniques utilisant des oligonucléotides dans la dystrophie musculaire de Duchenne. Il a souligné la 
nécessité d'améliorer l'homogénéité des patients inclus, ainsi que le choix des critères d'évaluation de l'efficacité des essais (test de marche de 6 minutes 6MWT par exemple). Helge Amthor (Université de Versailles Saint-Quentin-en Yvelines) a présenté le rôle de la myostatine, des protéines BMP et des facteurs de croissance TGF- $\beta$ sur la plasticité musculaire. Ana Buj-Bello (Généthon, Évry) a rapporté ses travaux de thérapie génique dans la myopathie myotubulaire liée à l'X qui ont permis une récupération rapide et à long terme (le recul est désormais de 3 ans) après une seule injection chez le chien modèle.

Dans l'après-midi, un moment fort a ponctué le congrès lors de la présentation des résultats d'essais cliniques menés chez des bébés atteints de SMA de type 1 par Richard Finkel (Philadelphie, USA) et Jerry Mendell (Colombus, USA). Des vidéos de bébés relevant leur tête et se tenant assis ou debout ont particulièrement ému l'assemblée. Deux stratégies thérapeutiques sont actuellement testées : un produit oligonucléotide antisens d'Ionis Pharmaceuticals (Californie, USA) et un produit de thérapie génique d'AveXis (Illinois, USA).

Le $5^{e}$ et dernier jour a été consacré aux projets de thérapies génique et cellulaire du muscle cardiaque, qu'il s'agisse de cardiomyopathies ou d'insuffisance cardiaque post-infarctus. Philippe Menasché (HEGP, Paris) qui mène le $1^{\text {er }}$ essai français utilisant des cellules souches embryonnaires chez des patients atteints d'infarctus (4 patients traités à ce jour) a souligné l'importance des facteurs trophiques apportés par les cellules greffées. Lucie Carrier (Hambourg, Allemagne) a présenté ses travaux chez la souris pour une thérapie génique d'une forme de cardiomyopathie hypertrophique liée aux mutations du gène MYBPC3. De son côté, Hélène Puccio (Illkirch, France) a rapporté de bons résultats de la thérapie génique chez la souris pour la cardiomyopathie de l'ataxie de Friedreich. Le vecteur utilisé (AAV10-FXN) est en cours de développement par la société Anapurna pour un essai chez l'Homme.

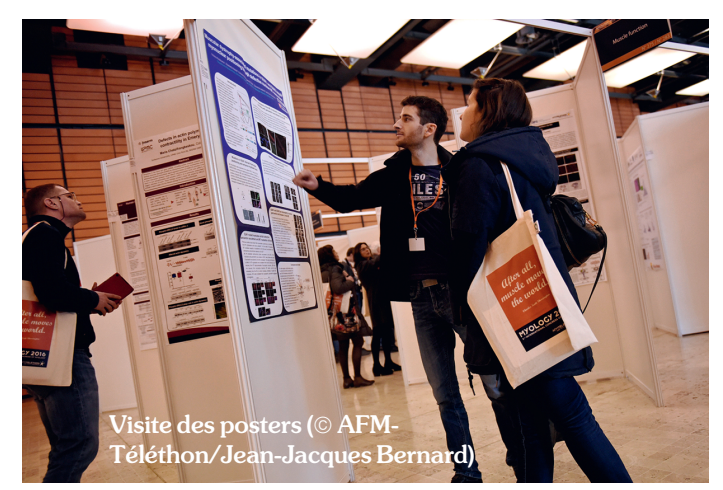

Cette $5^{e}$ édition du congrès a également permis aux jeunes chercheurs financés par l'AFM-Téléthon de présenter leurs travaux devant la communauté scientifique internationale. Deux d'entre eux ont reçu un prix pour leur présentation orale : Muriel Sébastien (Institut des neurosciences, Grenoble) et Pierre Klein (Institut de Myologie, Paris).

Enfin, à noter dans vos agendas, rendez-vous en 2019 pour la $6^{e}$ édition du congrès Myology !

International Congress of Myology: five days of unusual excitement

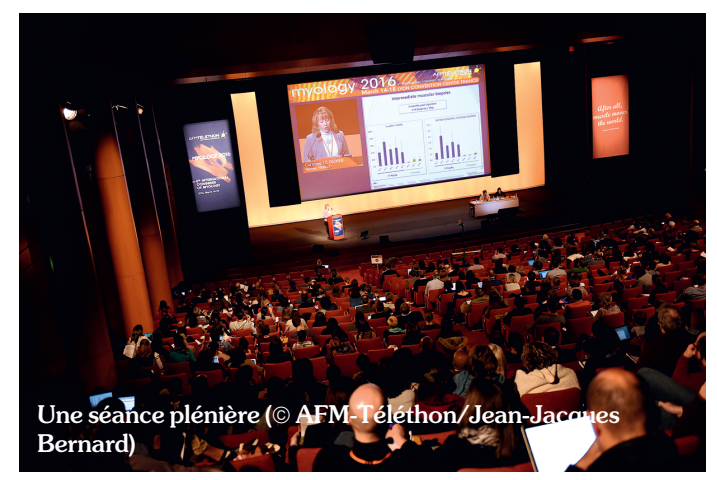

\section{LIENS D'INTÉRÊT}

Les auteurs déclarent n'avoir aucun lien d'intérêt concernant les données publiées dans cet article. 